\title{
The Fontan Operation: The Pursuit of Associated Lesions and Cumulative Trauma
}

\author{
Robert L. Hannan • Jennifer A. Zabinsky • Jane L. Salvaggio • Anthony F. Rossi • \\ Danyal M. Khan • Francisco A. Alonso • Jorge W. Ojito • David G. Nykanen • \\ Evan M. Zahn $\cdot$ Redmond P. Burke
}

Received: 17 May 2010/Accepted: 21 March 2011/Published online: 10 April 2011

(C) The Author(s) 2011. This article is published with open access at Springerlink.com

\begin{abstract}
Our programmatic approach to the Fontan operation has evolved to include using an extracardiac conduit with aggressive presumptive treatment of associated lesions either in the catheterization laboratory or the operating room. Fenestration is used selectively based on hemodynamics, anatomy, and presence of associated lesions. We reviewed our experience to determine the effectiveness and outcome of this strategy and to assess the cumulative trauma to the patients. The records of 137 consecutive patients who underwent Fontan at Miami Children's Hospital from 1995 to 2008 were reviewed. At mean follow up of 5.76 years, freedom from death or transplantation is $94.2 \%$ (129/137). Median age at operation was 4.6 years. Longer length of stay correlated with older operative age $(P=0.0056)$. Pacemakers were implanted in $11.7 \%$ (16/137). Additional (not pre-Glenn or pre-Fontan) interventional catheterizations were performed in $51.8 \%$ (71/137). Additional operations were done in $10.2 \%$ (14/ 137). No patient has required replacement or revision of the extracardiac conduit. Our current approach to the Fontan operation provides acceptable midterm results. The pursuit
\end{abstract}

R. L. Hannan ( $($ ) J J. A. Zabinsky · J. L. Salvaggio ·

F. A. Alonso - J. W. Ojito · R. P. Burke

Department of Cardiovascular Surgery, Congenital Heart

Institute at Miami Children's Hospital, 3100 SW 62nd Avenue,

Miami, FL 33155, USA

e-mail: rhannan001@aol.com

A. F. Rossi · D. M. Khan · E. M. Zahn

Department of Cardiology, Congenital Heart Institute at Miami Children's Hospital, 3100 SW 62nd Avenue, Miami, FL 33155, USA

D. G. Nykanen

Department of Pediatric Cardiology, Arnold Palmer Hospital for Children, Orlando, FL, USA of residual lesions results in a significant number of additional interventional catheterizations and operative procedures but might have an important influence on long-term survival after the Fontan procedure.

Keywords Cardiac surgery - Cardiac catheterization/ intervention $\cdot$ Congenital heart disease Pediatric

\section{Introduction}

Approaches to single ventricle palliation have evolved since the introduction of the Fontan operation in 1971 [4]. This evolution encompasses multiple technical and anatomic challenges. The technical approach to the Fontan operation itself has evolved into two major approaches: the lateral tunnel technique [3] and the extracardiac technique $[9,12]$. Bidirectional cavopulmonary shunt as an interim staging procedure [7] between initial palliation and Fontan is now widely used. Fenestration in the Fontan pathway [1] might reduce the risk of Fontan operation in some patients.

In addition to the technical evolution of the Fontan procedure, the patients undergoing Fontan operation have changed. Improved results in single ventricle palliation of all types and especially of hypoplastic left heart syndrome have changed the anatomic diagnosis of patients undergoing Fontan procedure, including the increasing proportion of patients with systemic right ventricles. The 10 Choussat criteria [2] are rarely present in a modern Fontan patient.

In addition to technical changes in the operation and a broadening of patients being offered the Fontan procedure, other important evolutions in cardiac care have occurred. A significant one is the improved ability to intervene on intracardiac and extracardiac lesions in the cardiac catheterization laboratory [11]. 
Our programmatic approach to the Fontan operation has evolved since 1995. We now perform almost exclusively extracardiac Fontan procedures using expanded polytetrafluoroethylene grafts from the inferior vena cava to the pulmonary artery-superior vena caval anastomosis. Fenestration is performed on a selective basis. Bidirectional cavopulmonary shunts are performed on all patients who are initially palliated as babies; some older children who do not require initial palliation might undergo primary Fontan. Since 2001, we have programmatically avoided deep hypothermic circulatory arrest at all stages of single ventricle palliation [5]. Both bidirectional cavopulmonary anastomosis and Fontan operations are performed with the heart warm and beating if possible. We aggressively address associated lesions on a presumptive basis with treatment either in the cardiac catheterization laboratory or the operating room.

We retrospectively reviewed our single-institution Fontan experience from November 1995 to January 2008 to evaluate the evolution in our approach to the Fontan operation, the outcome of our approach, and the intensity of effort and trauma to patients required to achieve these results.

\section{Materials and Methods}

The study was approved by the Western Institutional Review Board on February 22, 2008, and individual consent was waived. A retrospective study of 137 consecutive patients who underwent a Fontan procedure at Miami Children's Hospital from November 1995 to January 2008 was conducted. All medical records, including surgical and catheterization records, were reviewed. Cumulative trauma was defined as all operations, interventions, and hospitalizations to achieve orthoterminal correction with the Fontan operation. This included additional chest tubes, pacemakers, and operations in addition to the usual palliative procedures. It also included interventions in the cardiac catheterization laboratory, especially those done at a time separate from preoperative diagnostic catheterizations. Our current philosophy is to complete the Fontan operation at around 4 years of age or $15 \mathrm{~kg}$.

Data are given as means with standard deviations or medians with ranges, as appropriate. Mean values were compared for Normal distributions using the $t$-test, and median values were compared for non-Normal distributions with the Mann-Whitney rank sum test. The Fisher's exact test was used for categorical data. Correlations were analyzed with the Pearson product moment correlation test. Survival curves were created using the Kaplan-Meier method. All analyses were performed with SigmaStat ${ }^{\circledR}$ statistical software (version 3.1; Systat Software, Inc.,
Point Richmond, CA). All $P$-values less than 0.05 were considered statistically significant.

\section{Results}

The Fontan operation was performed at a median age of 4.6 years (range: 1.5-17.0) with a median weight of $16.5 \mathrm{~kg}$ (range: 9.4-54). Patients' diagnoses are summarized in Table 1 . For the majority of patients $(89.8 \%)$, the Fontan operation consisted of an expanded polytetrafluoroethylene with external reinforcement (Bard Peripheral Vascular Inc., Tempe, AZ) extracardiac conduit connecting the inferior vena cava to the superior vena cava-pulmonary artery junction. The size of the extracardiac conduit was either $16 \mathrm{~mm}(40 / 123)$ or $19 \mathrm{~mm}$ (81/123); two patients received $15-\mathrm{mm}$ and $20-\mathrm{mm}$ aortic homografts, respectively. Size was based on the weight of the patient and the size of the inferior vena cava intraoperatively. There was a significant difference in weight between patients with 16-mm extracardiac conduits and those with 19-mm conduits (median: $14.95 \mathrm{~kg}$ vs. $17.40 \mathrm{~kg} ; P \leq 0.001$ ) (Fig. 1).

Fifty percent $(69 / 137)$ of all patients underwent fenestration and the other half (68/137) were not fenestrated. The decision to fenestrate was a subjective one, based on systemic ventricle morphology, pulmonary artery anatomy and pressures, and the presence or absence of associated lesions. In general, patients with systemic right ventricles who had other lesions addressed at the time of the Fontan, such as aortic arch reconstruction, or who had elevated pulmonary artery pressures received fenestrations. Fenestration is performed by anastomosing the right atrium at the superior portion of the right atrial-inferior vena cava suture line to a 4- or 5-mm punch hole in the conduit. Intracardiac lateral tunnel Fontan was uncommon $(10.2 \%$; 14/137) and

Table 1 Diagnoses of patients who underwent Fontan

\begin{tabular}{lcc}
\hline Diagnosis & $n$ & $\%$ \\
\hline HLHS & 38 & 27.7 \\
Tricuspid atresia & 35 & 25.5 \\
TGA & 15 & 10.9 \\
DORV & 11 & 8.0 \\
Unbalanced AVC & 7 & 5.1 \\
PA-IVS & 7 & 5.1 \\
Mitral Atresia & 7 & 5.1 \\
Other $^{\mathrm{a}}$ & 17 & 12.4 \\
\hline
\end{tabular}

$A V C$ atrioventricular canal, DORV double-outlet right ventricle, HLHS hypoplastic left heart syndrome, PA-IVS pulmonary atresia with intact ventricular septum, $T G A$ transposition of the great arteries

a Other diagnoses include double inlet ventricle, single ventricle, tetralogy of Fallot/pulmonary atresia-ventricular septal defect, aortic stenosis, and tricuspid valve disease/Ebstein's anomaly 
Fig. 1 Extracardiac conduit size versus weight

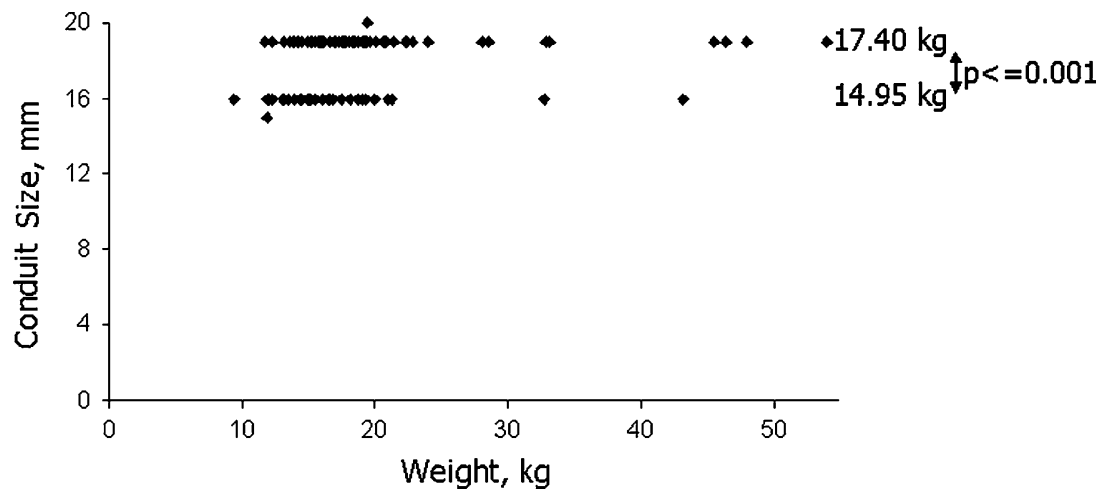

was performed more commonly early in the series: Only 1 of the last 113 patients had a lateral tunnel (Fig. 2). The lateral tunnel technique was done with fenestration.

The majority of patients $(94.2 \%$; 129/137) were first staged with a bidirectional cavopulmonary anastomosis, whereas $5.8 \%(8 / 137)$ underwent primary Fontan, with both the bidirectional cavopulmonary connection and the Fontan connection in the same procedure. At the time of the bidirectional cavopulmonary anastomosis, 24.5\% (27/110) of patients required cardioplegic arrest. This number excludes patients who had a primary Fontan or who underwent bidirectional cavopulmonary anastomosis at another institution. At the time of Fontan, 32.1\% (44/137) required cardioplegia. Of the patients who underwent extracardiac Fontan, circulatory arrest was used in $3.3 \%(4 / 123)$ at the time of bidirectional cavopulmonary anastomosis or Fontan, and cardioplegic arrest was used in $40.7 \%$ (50/123).

The median length of stay was 9.0 days (range: 4-76). Length of stay positively correlated with age at the time of the Fontan operation $(P=0.0056)$ (Fig. 3). The length of stay for patients with fenestrations was significantly longer, compared to those with nonfenestrated Fontans (median length of stay of 13 days for fenestrated patients vs. 9 days for nonfenestrated; $P=0.005$ ) (Fig. 3).

Kaplan-Meier survival was $95.6 \%$ and $93.6 \%$ at 5 and 10 years, respectively, with a mean follow-up of 5.76 years (Fig. 4). Freedom from mortality and transplantation was $94.2 \%(129 / 137)$.

Fig. 2 Type of Fontan procedure by date of surgery$$
\text { procedure by date of surgery }
$$

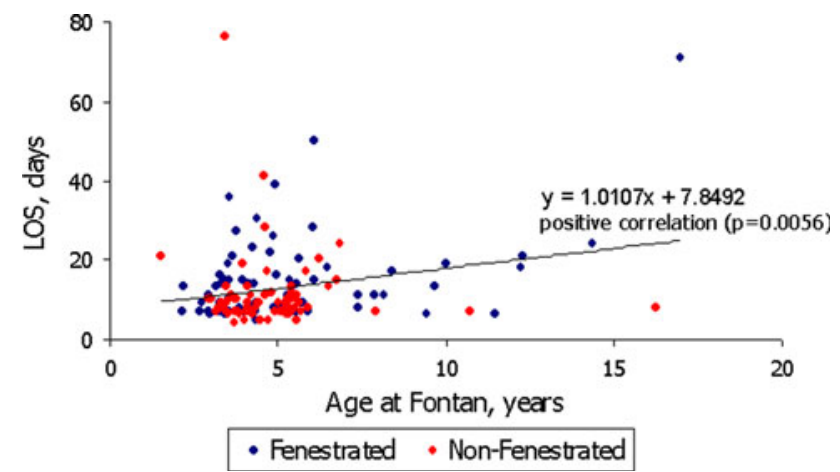

Fig. 3 Length of stay (LOS) versus age at time of Fontan

Catheter Interventions

Of this goup, $62.8 \%(86 / 137)$ underwent a total of 216 catheter interventions, excluding collaterals incidentally addressed at the time of the usual pre-bidirectional cavopulmonary anastomosis or pre-Fontan catheterization; $51.8 \%$ (71/137) required additional interventional catheterizations not coincident with the usual pre-bidirectional cavopulmonary anastomosis or pre-Fontan catheterizations (Fig. 5a). The most common catheter intervention was occlusion of collaterals. Excluding all collateral occlusions, more patients with fenestrated Fontans required catheter interventions compared to those without fenestration (66.7\% vs. $45.6 \% ; P=0.021)$.

More patients required angioplasty or stenting of the left pulmonary artery compared to the right pulmonary artery,

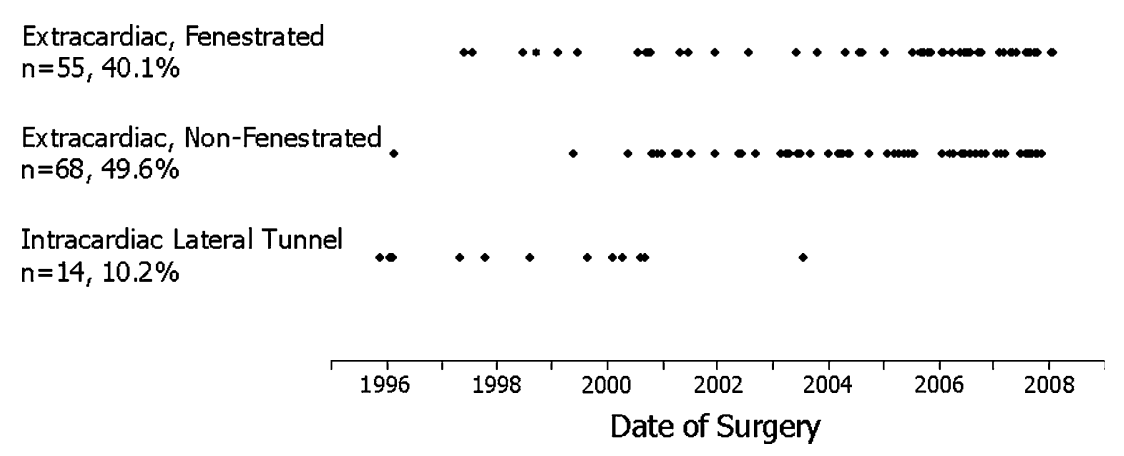




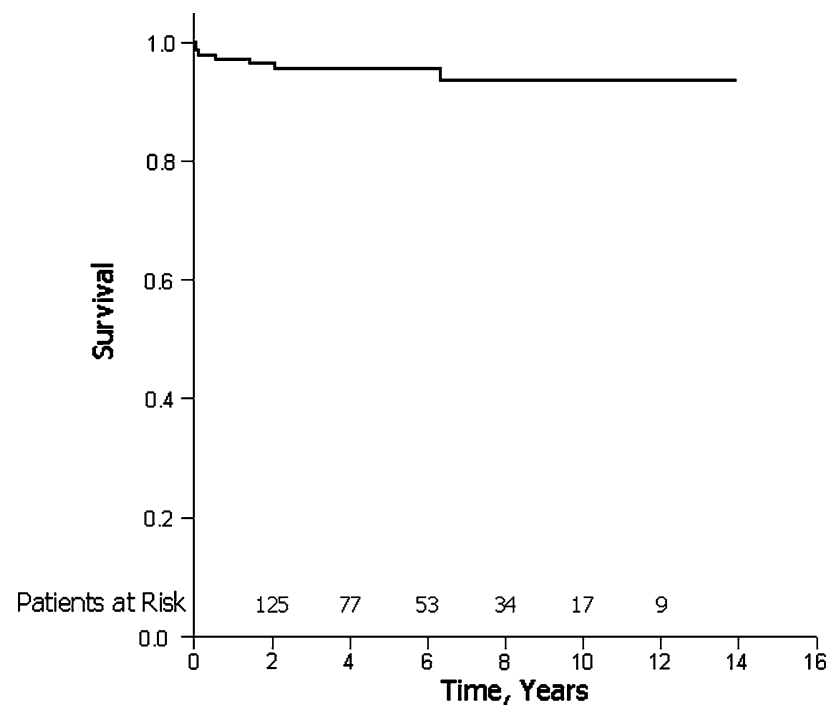

Fig. 4 Kaplan-Meier survival curve

both before and after the Fontan procedure (Table 2). The length of stay was significantly longer for those patients who had pre-Fontan catheter interventions on their left and/ or right pulmonary arteries (median length of stay of 13 days vs. 9 days for those without intervention on their pulmonary arteries; $P=0.031$ ). Of the patients who required catheter interventions post-bidirectional cavopulmonary anastomosis, $14.3 \%$ (5/35) underwent the intervention within the same hospitalization. For catheter interventions post-Fontan, $37.2 \%(16 / 43)$ of patients had the procedures during the same hospitalization.

Two patients $(1.5 \%)$ required fenestration creation and stent placement post-Fontan by catheterization. Three patients $(2.2 \%)$ required angioplasty and stenting of their existing fenestrations. For one patient, the fenestration was stented. One hybrid procedure was performed to create and stent a fenestration. Fourteen patients (10.2\%) underwent closure of their fenestrations in the catheterization laboratory (Table 2).

\section{Surgical Procedures}

A total of 206 surgical interventions were performed in 89 patients (65\%). Of these interventions, $14.6 \%(30 / 206)$ were pulmonary artery plasties at the time of bidirectional cavopulmonary anastomosis. Aside from the initial palliation, bidirectional cavopulmonary anastomosis, and Fontan operation, 14 patients (10.2\%) required at least 1 additional operation (Fig. 5b). No patient has required replacement or revision of the extracardiac conduit. Three patients had postoperative surgical fenestration creation or enlargement. Additionally, $17.5 \%$ of patients (24/137) required chest tubes postoperatively. There was no significant difference
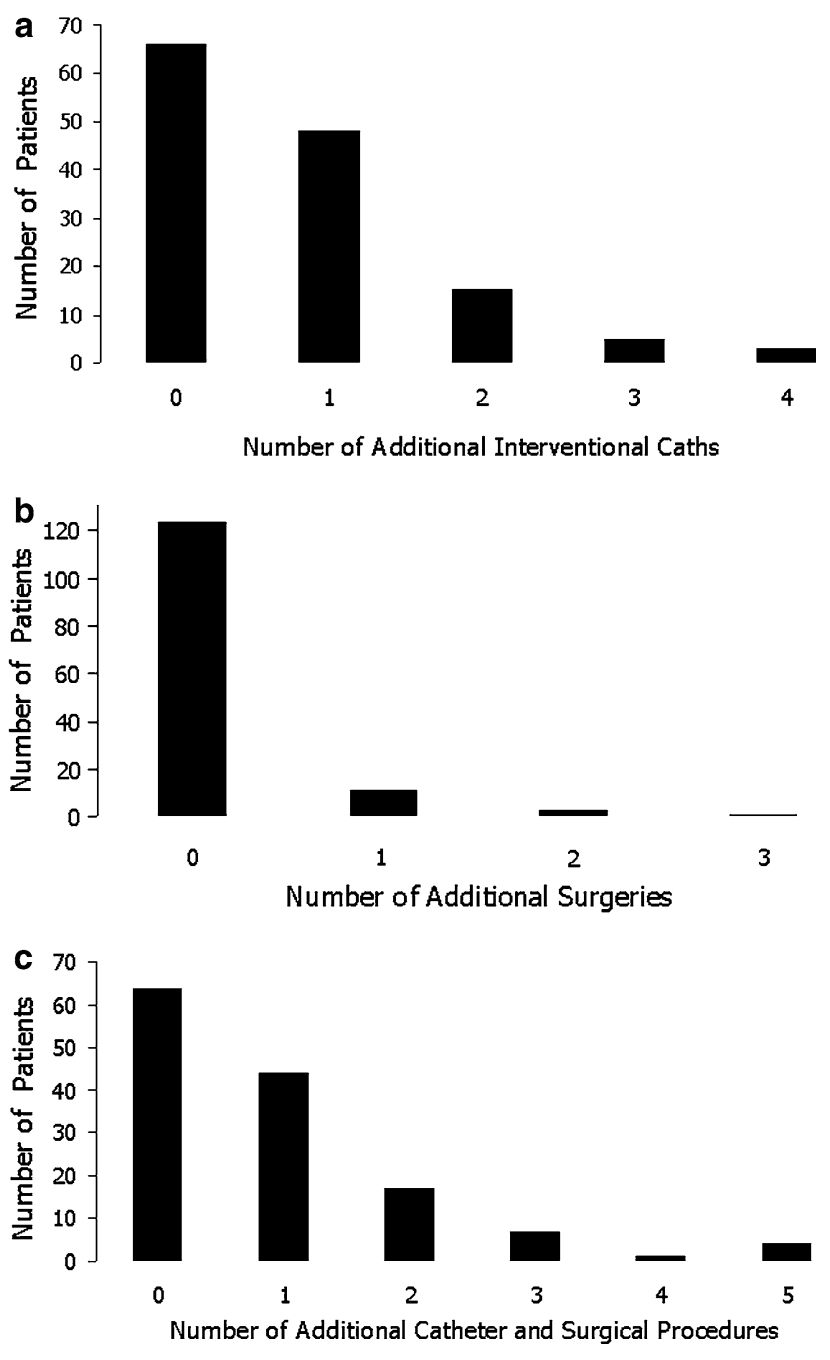

Fig. 5 Number of patients requiring a interventional catheterizations in addition to the pre-bidirectional cavopulmonary anastomosis and pre-Fontan catheterizations, $\mathbf{b}$ surgical procedures, in addition to an initial palliation, bidirectional cavopulmonary anastomosis, and Fontan, excluding the reinsertion of chest tubes, and $\mathbf{c}$ additional catheterizations or surgeries

in need for chest tubes between fenestrated Fontan procedures and nonfenestrated $(P=0.822)$.

Sixteen patients $(11.7 \%)$ required placement of a pacemaker, and for the majority (12/16), implantation was during the Fontan operation. Additionally, six patients had pacing leads placed either concomitantly $(n=5)$ or post-Fontan $(n=1)$. Two patients required ablation of arrhythmias.

Catheter, surgical, and hybrid interventions are summarized in Tables 3, 4, and 5, respectively. One hundred seventeen of 137 (85.4\%) patients required interventions at any point, either in the catheterization laboratory or the operating room. Four patients underwent intraoperative stent placement in the pulmonary arteries during the Fontan procedure. Seventy-three of 137 (53.3\%) patients required 
Table 2 Catheter interventions performed pre-Fontan and post-Fontan

\begin{tabular}{|c|c|c|c|c|c|}
\hline \multicolumn{3}{|c|}{ Pre-Fontan interventions } & \multicolumn{3}{|c|}{ Post-Fontan interventions } \\
\hline & No. of patients & $\%$ & & No. of patients & $\%$ \\
\hline Collateral occlusion & 46 & 33.6 & Collateral occlusion & 14 & 10.2 \\
\hline Aorta & 18 & 13.1 & Bilateral PAs & & \\
\hline Angioplasty & 18 & 13.1 & Angioplasty & 2 & 1.5 \\
\hline Stent & 2 & 1.5 & LPA & 13 & 9.5 \\
\hline Stent angioplasty & 2 & 1.5 & Angioplasty & 1 & 0.7 \\
\hline Bilateral PAs & & & Stent & 12 & 8.8 \\
\hline Angioplasty & 3 & 2.2 & Stent angioplasty & 1 & 0.7 \\
\hline LPA & 18 & 13.1 & RPA & 7 & 5.1 \\
\hline Angioplasty & 13 & 9.5 & Angioplasty & 1 & 0.7 \\
\hline Stent & 9 & 6.6 & Stent & 6 & 4.4 \\
\hline Stent Angioplasty & 1 & 0.7 & Fenestration & 6 & 4.4 \\
\hline RPA & 6 & 4.4 & Creation & 2 & 1.5 \\
\hline Angioplasty & 4 & 2.9 & Angioplasty & 3 & 2.2 \\
\hline Stent & 3 & 2.2 & Stent & 6 & 4.4 \\
\hline Stent angioplasty & 1 & 0.7 & Fenestration closure & 14 & 10.2 \\
\hline Atrial septostomy & 9 & 6.6 & & & \\
\hline
\end{tabular}

$L P A$ left pulmonary artery, $P A s$ pulmonary arteries, $R P A$ right pulmonary artery

Table 3 Pre-Fontan procedures

\begin{tabular}{lccl}
\hline Procedure & Catheter & Surgical & Hybrid \\
\hline Aorta & 18 & 6 & 1 \\
Pulmonary arteries & 24 & 33 & 2 \\
Pulmonary valvuloplasty & 2 & 0 & 0 \\
Atrial septostomy/septectomy & 9 & 18 & 3 \\
Pacemaker & - & 3 & 0 \\
\hline
\end{tabular}

additional catheterizations or operations beyond initial palliation, pre-bidirectional cavopulmonary anastomosis catheterization, bidirectional cavopulmonary anastomosis, pre-Fontan catheterization, and Fontan procedure (Fig. 5c).

\section{Mortality}

There were seven mortalities (5.1\%); two were in-hospital deaths, after undergoing Fontan operation at 6 years and 12 years of age, respectively. One patient died from a bronchial hemorrhage and the second death was due to plastic bronchitis. The remaining five mortalities were after discharge at a range of 16 days to 6.4 years post-Fontan operation. One patient died as an outpatient from pericardial effusion. Another suffered from protein-losing enteropathy. One patient died after attempted two-ventricle conversion at another institution. Two patients had neurological damage prior to death. Two Fontans were taken down; one of these patients was among the seven mortalities and the other went on to heart transplantation.
Table 4 Intraoperative procedures during Fontan

\begin{tabular}{lcl}
\hline Procedure & Surgical & Hybrid \\
\hline Aorta & 6 & 0 \\
Tricuspid or mitral valvuloplasty & 4 & 0 \\
Pulmonary arteries & 14 & 4 \\
Atrial septectomy & 11 & 0 \\
Pacemaker & 12 & 0 \\
\hline
\end{tabular}

Table 5 Post-Fontan procedures

\begin{tabular}{lccl}
\hline Procedure & Catheter & Surgical & Hybrid \\
\hline Pulmonary arteries & 15 & 0 & 0 \\
Fontan conduit & 5 & 0 & 0 \\
Fenestration creation/stenting & 6 & 3 & 1 \\
Fenestration closure & 14 & 0 & 0 \\
Pacemaker & - & 1 & 0 \\
Tube thoracostomy & - & 24 & 0 \\
Fontan takedown & - & 2 & 0 \\
\hline
\end{tabular}

\section{Discussion}

Our current approach to the Fontan procedure offers acceptable early mortality and morbidity compared to literature reports $[6,8,13]$. Postoperative length of stay and need for reinsertion of chest tubes for postoperative effusions are comparable to literature reports $[10,13]$. No 
patient required revision of the extracardiac conduit because of growth or for other reasons. Four patients (2.9\%) required stenting of the Fontan conduit, and one patient underwent angioplasty of the conduit.

Our approach to bidirectional cavopulmonary anastomosis and extracardiac Fontan has resulted in an acceptable rate of pacemaker implantation (11.7\%). In addition, at our relatively short follow-up, arrhythmias requiring ablation have occurred in only two patients.

The approach to fenestration appears to have been successful and validated. The need to create a fenestration postoperatively has been uncommon $(5 / 137 ; 3.6 \%)$. The length of stay for fenestrated Fontans was significantly longer than nonfenestrated, indicating that we have successfully identified a high-risk group. Significantly more patients with fenestration required catheter interventions (66.7\%), compared to those without fenestration (45.6\%; $P=0.021$ ). However, there was no significant difference for reinsertion of chest tubes, pacemaker implantation, and mortality between fenestrated and nonfenestrated patients. Twenty percent of fenestrated patients underwent fenestration closure in the catheterization laboratory.

The technique for fenestration has evolved. We now sew the anterior portion of the atrial transection directly to a 4-mm punch hole in the anterior medial surface of the graft. We have not systematically studied fenestration patency, but this technique appears to be superior to others.

Our presumptive approach to treating associated lesions results in a significant number of interventions: 216 in the catheterization laboratory and 206 in the operating room. Many of these interventions are, however, associated with the usual preoperative catheterizations or the operative procedure itself (Fig. 5). Many of the operative interventions were pulmonary artery angioplasties at the time of the bidirectional cavopulmonary anastomosis. Additional catheterizations for intervention were required in 71 patients $(51.8 \%)$ and additional operations were required in 14 patients $(10.2 \%)$. Forty-four $(32.1 \%)$ patients required only one extra catheter or surgical procedure.

\section{Limitations}

Our study is clearly limited by our inability to directly correlate our approach to associated lesions and our outcomes. Although we might infer that this part of our overall Fontan strategy has an important role in our outcomes, such inference might be incorrect. It might be just as fruitful to wait until associated lesions have important consequences before treatment. We find this approach unpalatable, in part, because of the fragility of Fontan patients with residual lesions in the early postoperative period.

A significant limitation of our study is our limited period of follow-up and the limitations it imposes on long-term conclusions. A possible limitation of the extracardiac Fontan operation is the use of a circumferential artificial conduit, potentially requiring reoperation as patients reach adulthood. We currently have not observed the need for conduit replacement in any patient.

The Fontan circulation is a life-long disease state with its own associated lesions. The long-term incidence of important sequelae, such as arrhythmia and protein-losing enteropathy, is not addressed in this report. We would propose, however, that good short-term outcomes might positively affect long-term outcomes.

Open Access This article is distributed under the terms of the Creative Commons Attribution Noncommercial License which permits any noncommercial use, distribution, and reproduction in any medium, provided the original author(s) and source are credited.

\section{References}

1. Bridges ND, Lock JE, Castaneda AR (1990) Baffle fenestration with subsequent transcatheter closure. Modification of the Fontan operation for patients at increased risk. Circulation 82:1681-1689

2. Choussat A, Fontan F, Besse P, Vallot F, Chauve A, Bricaud H (1978) Selection criteria for Fontan's procedure. In: Anderson RH, Shinbourne EA (eds) Pediatric Cardiology. Churchill Livingstone, Edinburg

3. d'Udekem Y, Iyengar AJ, Cochrane AD, Grigg LE, Ramsay JM, Wheaton GR, Wheaton GR, Penny DJ, Brizard CP (2007) The Fontan procedure: contemporary techniques have improved longterm outcomes. Circulation 116:I157-I164

4. Fontan F, Baudet E (1971) Surgical repair of tricuspid atresia. Thorax 26:240-248

5. Hannan RL, Ybarra MA, Ojito JW, Alonso FA, Rossi AF, Burke RP (2006) Complex neonatal single ventricle palliation using antegrade cerebral perfusion. Ann Thorac Surg 82:1278-1284

6. Hirsch JC, Goldberg C, Bove EL, Salehian S, Lee T, Ohye RG, Devaney EJ (2008) Fontan operation in the current era: a 15-year single institution experience. Ann Surg 248:402-410

7. Hopkins RA, Armstrong BE, Serwer GA, Peterson RJ, Oldham HN Jr (1985) Physiological rationale for a bidirectional cavopulmonary shunt. A versatile complement to the Fontan principle. J Thorac Cardiovasc Surg 90:391-398

8. Kim SJ, Kim WH, Lim HG, Lee JY (2008) Outcome of 200 patients after an extracardiac Fontan procedure. J Thorac Cardiovasc Surg 136:108-116

9. Nakano T, Kado H, Tachibana T, Hinokiyama K, Shiose A, Kajimoto M, Ando Y (2007) Excellent midterm outcome of extracardiac conduit total cavopulmonary connection: results of 126 cases. Ann Thorac Surg 84:1619-1625

10. Pace NC, Oppido G, Angeli E, Giardini A, Resciniti E, Gargiulo G (2009) Results of the modified Fontan procedure are not related to age at operation. Eur J Cardiothorac Surg 37:645-650

11. Stanfill R, Nykanen DG, Osorio S, Whalen R, Burke RP, Zahn EM (2008) Stent implantation is effective treatment of vascular stenosis in young infants with congenital heart disease: acute 
implantation and long-term follow-up results. Catheter Cardiovasc Intervent 71:831-841

12. Tokunaga S, Kado H, Imoto Y, Masuda M, Fukae K, Shiokawa Y, Fusazaki N, Ishikawa S, Yasui H (2002) Total cavopulmonary connection with an extracardiac conduit: experience with 100 patients. Ann Thorac Surg 73:76-80
13. Tweddell JS, Nersesian M, Mussatto KA, Nugent M, Simpson P, Mitchell ME, Ghanayem NS, Pelech AN, Marla R, Hoffman GM (2009) Fontan palliation in the modern era: factors impacting mortality and morbidity. Ann Thorac Surg 88:1291-1299 